\title{
Neoadjuvant Nivolumab and Chemotherapy in Patients with Locally Advanced Non-Small Cell Lung Cancer: A Retrospective Study
}

\author{
Hengyu Zhai, Wenhai Li, Kun Jiang, Yanan Zhi, Zhao Yang \\ Department of Thoracic Surgery, Xi'an International Medical Center Hospital, Xi'an, Shaanxi, 7I0000, People's Republic of China
}

Correspondence: Zhao Yang, Department of Thoracic Surgery, Xi'an International Medical Center Hospital, No. 777 Xitai Road, Xi'an, Shaanxi, 710000, People's Republic of China, Email Zhaoyang_666@aliyun.com

Background: Patients with locally advanced (stage III) non-small cell lung cancer (NSCLC) demonstrate broad anatomic heterogeneity with modest survival benefits. Immune checkpoint inhibitors (ICIs) have shown survival benefit in metastatic NSCLC. We conducted this study to evaluate the efficacy and safety of neoadjuvant nivolumab in combination with chemotherapy in the treatment of this population.

Methods: We retrospectively evaluated patients with locally advanced NSCLC receiving neoadjuvant nivolumab and chemotherapy (paclitaxel with carboplatin) followed by surgery at our institution from January 2019 to January 2020.

Results: A total of 46 eligible patients, 26 males, and 20 females were diagnosed with NSCLC in a stage IIIA (30 cases) and IIIB (16 cases) to receive neoadjuvant treatment. The treatment was well tolerated with just $7(15.2 \%)$ typical immune-related adverse events (hyperthyroidism, hyperglycemia, and rash) recorded. A total of 45 patients underwent surgical resection, and 43 (95.6\%) of them achieved a R0 resection. No major surgical complications were observed. There was a complete response (CR), partial response (PR), stable disease (SD), and progressive disease (PD) in 2 (4.3\%), 26 (56.5\%), 17 (37.0\%), 6 (26.1\%), and 1 (2.2\%) patients. Eight patients resulted in a major pathological response (MPR) (17.4\%) and 24 patients had a pathological complete response (pCR) (52.2\%). At the time of data cutoff (June 1, 2021), the median follow-up period was 15.5 months (IQR 3.9-29) and 27 (60\%) of 45 patients who had tumor resection were progression free. At 24 months, progression-free survival was $45.8 \%$ and overall survival was $79.9 \%$.

Conclusion: Nivolumab plus paclitaxel and carboplatin could be a potential neoadjuvant regimen for patients with locally advanced NSCLC, rendering a potentially lethal disease to one that is curable.

Keywords: non-small cell lung cancer, neoadjuvant therapy, immunotherapy, chemotherapy, DFS, OS

\section{Introduction}

Non-small cell lung cancer (NSCLC) accounts for approximately $80 \%$ of all lung cancer cases. ${ }^{1}$ Radical surgery remains the cornerstone of therapy in early-stage NSCLC (stage I-IIIA) with a fair 5-year survival; however, 30-60\% of these patients will develop recurrence or metastasis after surgery. ${ }^{2,3}$ In recent years, a large number of studies have explored neoadjuvant treatments for NSCLC, which demonstrated decent efficacy to reduce the probability of recurrence and prolong survival. ${ }^{4,5}$ A meta-analysis showed that NSCLC patients receiving neoadjuvant chemotherapy yielded an absolute improvement in 5-year overall survival by $5 \%$ (from $40 \%$ to $45 \%$ ). ${ }^{6}$ In this context, more promising neoadjuvant strategies are strongly required for patients with locally advanced NSCLC.

Recently, immune checkpoint inhibitors (ICIs) have revolutionized the non-small-cell lung cancer treatment landscape. The CheckMate and KEYNOTE-trials have shown several inhibitors blocking PD-(L)1 immune checkpoints as

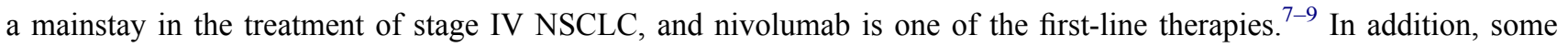
trials have reported the efficacy of PD-1 blockade plus chemotherapy in the treatment of advanced NSCLC. ${ }^{10-13}$ These encouraging results have consolidated the theoretical basis for the exploration of ICIs and chemotherapy in the 
neoadjuvant treatment of NSCLC. For instance, in CheckMate-159 trial, neoadjuvant administration of two doses of nivolumab resulted in a major pathological response (MPR) of $45 \%$ and pathological complete response (pCR) of $10 \%$ in resectable stage I-IIIA NSCLC patients. ${ }^{14}$ Gao et al. also demonstrated the therapeutic efficacy of neoadjuvant PD-1 inhibitor (Sintilimab) in patients with resectable NSCLC. ${ }^{15}$ The NADIM trial explored the anti-tumor activity and safety of neoadjuvant nivolumab combined with standard chemotherapy for resectable stage IIIA NSCLC. ${ }^{16}$ Among the 46 patients who received combined neoadjuvant therapy, the progression-free survival was $77.1 \%$ at 24 months; and 41 (89\%) patients underwent tumor resection with a MPR of $20 \%$ and pCR of $63 \%$. Recently, several retrospective studies reported that induction ICI-chemotherapy may be a valid treatment in patients with resectable or locally advanced NSCLC. ${ }^{17-19}$

Our study was aimed to retrospectively evaluate the feasibility, safety, and survival outcomes of neoadjuvant nivolumab in combination with standard chemotherapy for patients with locally advanced (stage IIIA-IIIB) NSCLC patients in the real-world setting.

\section{Materials and Methods}

\section{Patient Selection}

The study was approved by institutional review board of the Xi'an International Medical Center Hospital (201901-KY -06) and carried out in accordance with the Declaration of Helsinki (as revised in 2013). Written informed consent was obtained from all patients. We retrospectively enrolled and evaluated patients with locally advanced NSCLC (stage IIIAIIIB according to the 8th edition TNM staging of lung cancer of the American Joint Committee on Cancer) ${ }^{20}$ receiving a neoadjuvant nivolumab and chemotherapy followed by surgery at the Xi' an International Medical Center Hospital from January 2019 to January 2020. The inclusion criteria were as follows: (1) Aging 18 years or older with an Eastern Cooperative Oncology Group (ECOG) performance status (PS) of 0 or 1; (2) adequate organ function and could undergo surgical resection; (3) the clinical stage of all patients was confirmed based on the results of positron emission tomography with computed tomography (PET-CT), contrast enhanced CT, and magnetic resonance imaging (MRI) before neoadjuvant therapy; (4) with three cycles of nivolumab $(360 \mathrm{mg})$ on day 1 of each 21-day cycle. The exclusion criteria were as follows: (1) with other anti-tumor treatments before admission; (2) with other significant concurrent malignant tumors; (3) with known epidermal growth factor receptor (EGFR) mutations or anaplastic lymphoma kinase (ALK) rearrangements. Standard molecular testing was offered for patients before receiving immunotherapy.

\section{Neoadjuvant and Surgical Procedures}

Patients received the following drugs intravenously as neoadjuvant treatment: nivolumab (360 mg), paclitaxel $\left(200 \mathrm{mg} / \mathrm{m}^{2}\right.$ ), and carboplatin (area under the curve $6 ; 6 \mathrm{mg} / \mathrm{mL}$ per $\mathrm{min}$ ) on day 1 of each 21-day cycle, for three cycles before undergoing surgical resection. Contrast-enhanced CT of the chest was repeated to assess tumor response within 7 days before surgery. Surgery was scheduled 4-6 weeks after completion of neoadjuvant chemoimmunotherapy. All surgical resections were performed with video-assisted thoracoscopic surgery or thoracotomy based on standard institutional procedures. The surgical indicators, including extent of surgery, operative time, bleeding, hospital stay, and resection margin were first recorded. The postoperative morbidity, mortality, and surgical complications were also recorded.

\section{Endpoints of the Study}

The primary endpoint was disease-free survival (DFS) and overall survival (OS) at 24 months. DFS was defined as the time from diagnosis until disease recurrence or death, and overall survival defined as the time from diagnosis to date of death. Secondary endpoints were radiologic response, MPR and pCR, and adverse events (AEs). Radiologic responses were assessed according to Response Evaluation Criteria in Solid Tumors (RECIST) version 1.1. AEs were graded according to the NCI-CTCAE (version 4.0). MPR was defined as $10 \%$ or fewer residual tumor cells at the time of surgery, and pCR was defined as tumors without any viable tumor cells. 


\section{Statistical Analyses}

All statistical analyses were done using SPSS 20.0 (IBM SPSS Inc., Chicago, IL, USA). Continuous variables were presented as a mean \pm standard deviation or median and range when appropriate, while categorical variables were expressed as counts and percentages. The Chi-square test or Fisher's exact test were used to compare categorical variables. The Kaplan-Meier method was used to estimate DFS and OS and corresponding 95\% CIs. Differences were considered to be significant when $P<0.05$.

\section{Results}

\section{Patient Characteristics}

Between January 1, 2019, and January 1, 2020, 46 eligible patients were enrolled in this study. The baseline characteristics are presented in Table 1. There were 26 (56.5\%) males and 20 (43.5\%) females, and only $3(6.5 \%)$ patients never smoked. Seventeen (37.0\%) of 46 patients had ECOG PS 0, and 29 (63.0\%) had ECOG PS 1. Twenty-seven (58.7\%) of 46 patients had squamous carcinoma, and 19 (41.3\%) had adenocarcinoma. Thirty (65.2\%) patients had stage IIIA and 16 $(34.8 \%)$ had stage IIIB. Tumor PD-L1 expression at baseline was $1 \%$ or lower in $16(34.8 \%)$ patients and $50 \%$ or higher in $12(26.1 \%)$ patients. After receiving the neoadjuvant chemo-immunotherapy, 45 patients underwent surgical resection.

Table I Patient Characteristics

\begin{tabular}{|c|c|}
\hline Characteristics & Patients $(n=46)$ \\
\hline Age & $63(56-73)$ \\
\hline \multicolumn{2}{|l|}{ Sex } \\
\hline Male & $26(56.5 \%)$ \\
\hline Female & $20(43.5 \%)$ \\
\hline \multicolumn{2}{|l|}{ History of smoking } \\
\hline Current/ex-smoker & $43(93.5 \%)$ \\
\hline Never-smoker & $3(6.5 \%)$ \\
\hline \multicolumn{2}{|l|}{ ECOG PS } \\
\hline 0 & $17(37.0 \%)$ \\
\hline I & $29(63.0 \%)$ \\
\hline BMI, $\mathrm{kg} / \mathrm{m}^{2}$ & $24.51(24.12-28.62)$ \\
\hline \multicolumn{2}{|l|}{ Pathological type } \\
\hline Squamous carcinoma & 27 (58.7\%) \\
\hline Adenocarcinoma & $19(41.3 \%)$ \\
\hline \multicolumn{2}{|l|}{ Clinical T stage } \\
\hline TI & 4 \\
\hline $\mathrm{T} 2$ & 11 \\
\hline $\mathrm{T} 3$ & 16 \\
\hline $\mathrm{T} 4$ & 15 \\
\hline \multicolumn{2}{|l|}{ Clinical $N$ stage } \\
\hline No & 10 \\
\hline $\mathrm{NI}$ & 15 \\
\hline N2 & 21 \\
\hline \multicolumn{2}{|l|}{ Clinical stage } \\
\hline IIIA & $30(65.2 \%)$ \\
\hline IIIB & $16(34.8 \%)$ \\
\hline \multicolumn{2}{|l|}{ PD-LI expression } \\
\hline$<1 \%$ & $16(34.8 \%)$ \\
\hline $\mathrm{I}-50 \%$ & $18(39.1 \%)$ \\
\hline$\geq 50 \%$ & $12(26.1 \%)$ \\
\hline Adjuvant nivolumab (at least I cycle) & $34(73.9 \%)$ \\
\hline
\end{tabular}

Abbreviations: BMI, body mass index; PD-LI, programmed death-ligand I. 
One patient did not receive surgery due to brain metastases. Thirty-four (73.4\%) patients received at least one cycle of adjuvant nivolumab.

\section{Surgical Treatment}

The surgical details are reported in Table 2. Of these 45 patients, 29 (64.4\%) had video-assisted thoracoscopic surgery and $16(35.6 \%)$ had a thoracotomy; $32(71.1 \%)$ patients underwent lobectomy, 10 (22.2\%) patients underwent bilobectomy, and $3(6.7 \%)$ patients underwent pneumonectomy. Forty-three (95.6\%) patients achieved a R0 resection and 2 (4.4\%) patients had a R1 resection. The median operation time was 220 minutes (IQR 70-360), the median amount of blood lost was $190 \mathrm{~mL}$ (IQR 60-560), and the median hospital stay was 8 days (IQR 4-18). There was no 30- or 90-day mortality or any other serious surgical complications. No surgical complications were related to neoadjuvant treatment.

Table 2 Surgical Procedures

\begin{tabular}{|l|l|}
\hline Characteristics & Patients (n = 45) \\
\hline Type of surgery & \\
Video-assisted thoracoscopic surgery & $29(64.4 \%)$ \\
Thoracotomy & $16(35.6 \%)$ \\
Surgical resection & \\
Lobectomy & $32(71.1 \%)$ \\
Bilobectomy & $10(22.2 \%)$ \\
Pneumonectomy & $3(6.7 \%)$ \\
Operation time, min & $220(70-360)$ \\
Bleeding, mL & $190(60-560)$ \\
Hospital stay, days & $8(4-18)$ \\
Resection margins & \\
R0 & $43(95.6 \%)$ \\
RI & $2(4.4 \%)$ \\
R2 & $0(0 \%)$ \\
Surgical complications & \\
Intraoperative blood transfusion & $4(8.9 \%)$ \\
$30-$ and 90-day mortality & $0(0 \%)$ \\
Postoperative arrhythmia & $1(2.2 \%)$ \\
Urinary tract infection or urinary retention & $2(4.4 \%)$ \\
\hline
\end{tabular}

Table 3 Radiologic Responses and Pathologic Responses

\begin{tabular}{|l|l|}
\hline Characteristics & Patients (n = 46) \\
\hline Radiologic responses & \\
Complete response & $2(4.3 \%)$ \\
Partial response & $26(56.5 \%)$ \\
Stable disease & $17(37.0 \%)$ \\
Progression disease & $1(2.2 \%)$ \\
Pathologic responses & \\
Incomplete pathological response & $14(30.4 \%)$ \\
Major pathological response & $8(17.4 \%)$ \\
Complete pathological response & $24(52.2 \%)$ \\
Pathological T down-staging & $36(78.2 \%)$ \\
Pathological N down-staging (to NI or N0) & $30(65.2 \%)$ \\
\hline
\end{tabular}




\section{Radiologic and Pathologic Responses}

The radiologic and pathologic responses are summarized in Table 3. Of all 46 patients, 2 (4.3\%) patients had a complete response (CR), 26 (56.5\%) patients had a partial response (PR), 17 (37.0\%) had a stable disease (SD), and 1 (2.2\%) had a progressive disease (PD). According to postoperative pathological results, $8(17.4 \%)$ patients who underwent surgery had a MPR, 24 (52.2\%) patients had a pCR, and 14 (30.4\%) patients had incomplete pathological response. Thirty-six (78.2\%) patients achieved pathological $\mathrm{T}$ downstaging and $30(65.2 \%)$ patients attained pathological $\mathrm{N}$ downstaging.

\section{Survival Outcomes}

At the time of data cutoff (June 1, 2021), 27 (60\%) of 45 patients who had tumor resection were alive without recurrence, with a median follow-up of 15.5 months (IQR 3.9-29). One (2.2\%) patient who did not undergo surgery had disease progression and died; 18 (39.1\%) patients who underwent surgery had disease progression, of whom 5 (10.9\%) had died. Detailed disease progression sites are shown in Table S1, with 7 (38.9\%) cases with cervicothoracic lymph node, 6 (33.3\%) with lung recurrence, 2 (11.1\%) with pleural dissemination, 2 (11.1\%) with bone metastases, and $1(5.6 \%)$ with brain metastases. In the entire patient cohort, the median DFS was 21.3 months and the median OS was not reached (Figure 1). The DFS rate was $67.0 \%$ at 12 months, $53.4 \%$ at 18 months, and $45.8 \%$ at 24 months; the OS rate was $90.5 \%$ at 12 months, $86.6 \%$ at 18 months, and $79.9 \%$ at 24 months (Figure 1).

\section{Adverse Events}

The AEs in detail are shown in Table 4. Of all 46 patients, 38 (82.6\%) had treatment-related AEs of grade 1 or 2 during neoadjuvant treatment and $9(19.6 \%)$ had AEs of grade 3 or worse. The most common treatment-related grade 1 or 2 AEs were leukopenia (13 [28.3\%]), neutropenia (13 [28.3\%]), thrombocytopenia (11 [23.9\%]), vomiting (10 [21.7\%]), fatigue (15 [32.6\%]), and alopecia (14 [30.4\%]). The most common treatment-related grade 3 or worse AEs included anorexia (2 [4.3\%]), vomiting (1 [2.2\%]), fatigue (3 [6.5\%]), alopecia (2 [4.3\%]), and arthralgia or myalgia (1 [2.2\%]). Seven (15.2\%) patients had typical immune-related AEs including grade 1-2 hyperthyroidism, hyperglycemia, and rash. No treatment-related deaths were reported.

\section{Subgroup Analysis}

In subgroup analyses by pathological response, patients with a MPR had better DFS $(P=0.005$, Figure $2 \mathrm{~A})$ and $\mathrm{OS}(P=$ 0.017 , Figure 2B) than those with incomplete pathological response. Moreover, optimal DFS $(P<0.001$, Figure $2 \mathrm{C})$ and OS $(P=0.010$, Figure 2D) were determined in patients with a pCR compared with those with incomplete pathological response. No differences in DFS $(P=0.870$, Figure $3 \mathrm{~A})$ and OS $(P=0.453$, Figure $3 \mathrm{~B})$ were observed between patients with PD-L1 positive or negative. As shown in Table S2, no significant association was found between PD-L1 status and radiological or pathological responses. In terms of adjuvant nivolumab, no significant survival benefits regarding DFS $(P=0.926$, Figure 3C) and OS $(P=0.278$, Figure 3D) were obtained in patients receiving adjuvant nivolumab.

A

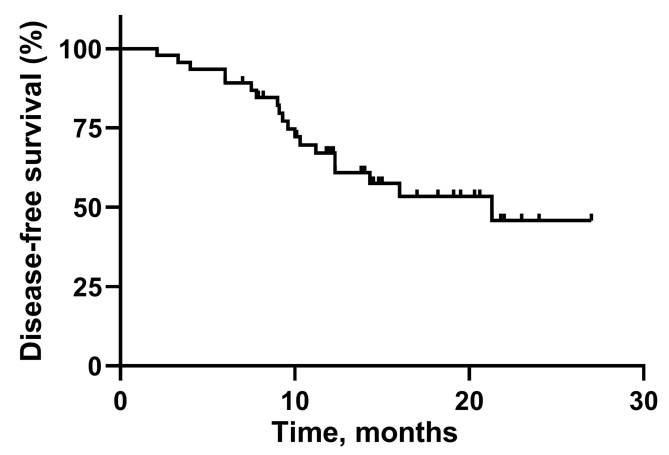

Number at risk 46

30 9
B

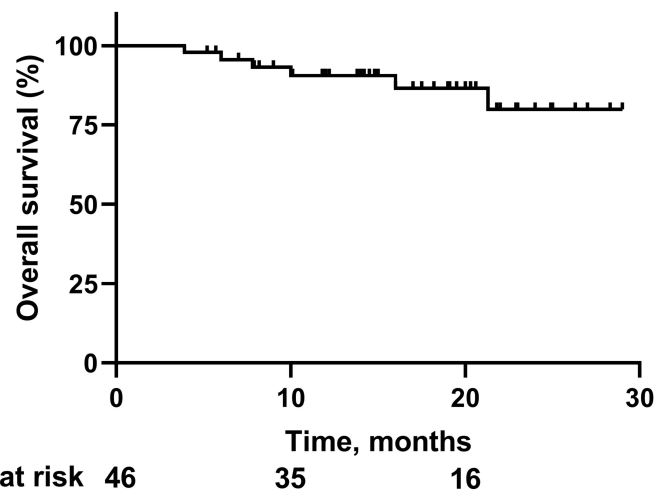

Figure I Kaplan-Meier survival curves in all patients. (A) Disease-free survival. (B) Overall survival. 
Table 4 Treatment-Related Adverse Events During Neoadjuvant Treatment $(n=46)$

\begin{tabular}{|l|c|l|l|}
\hline & Grade I-2 & Grade 3 & Grade 4 \\
\hline Any treatment-related adverse event & $38(82.6 \%)$ & $9(19.6 \%)$ & \\
Anemia & $6(13.0 \%)$ & & \\
Leukopenia & $13(28.3 \%)$ & & \\
Neutropenia & $13(28.3 \%)$ & & \\
Thrombocytopenia & $11(23.9 \%)$ & $2(4.3 \%)$ & \\
Anorexia & $12(6.7 \%)$ & $1(2.2 \%)$ & \\
Vomiting & $10(21.7 \%)$ & & \\
Diarrhea & $5(10.9 \%)$ & $3(6.5 \%)$ & \\
Constipation & $3(6.5 \%)$ & $2(4.3 \%)$ & \\
Fatigue & $15(32.6 \%)$ & & \\
Alopecia & $14(30.4 \%)$ & & \\
Hypothyroidism & $3(6.5 \%)$ & & \\
Rash & $2(4.3 \%)$ & $2(4.3 \%)$ & \\
Hyperglycemia & $3(6.5 \%)$ & & \\
Arthralgia or myalgia & & & \\
\hline
\end{tabular}

\section{Discussion}

This is the first observational study to test the safety, feasibility, antitumor activity, and survival outcomes of neoadjuvant nivolumab combined with chemotherapy for locally advanced NSCLC patients (stage IIIA-B) in a real-world setting. Our study yielded promising results with a DFS of $45.8 \%$ and an OS of $79.9 \%$ at 24 months. It is also encouraging for patients to achieve a MPR of $17.4 \%$ and a pCR of $52.2 \%$. The neoadjuvant treatment was safe and well tolerated with reasonable AEs.

A

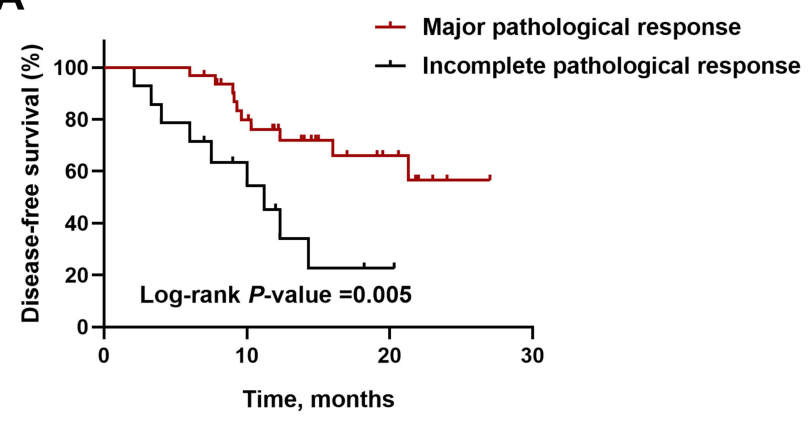

C

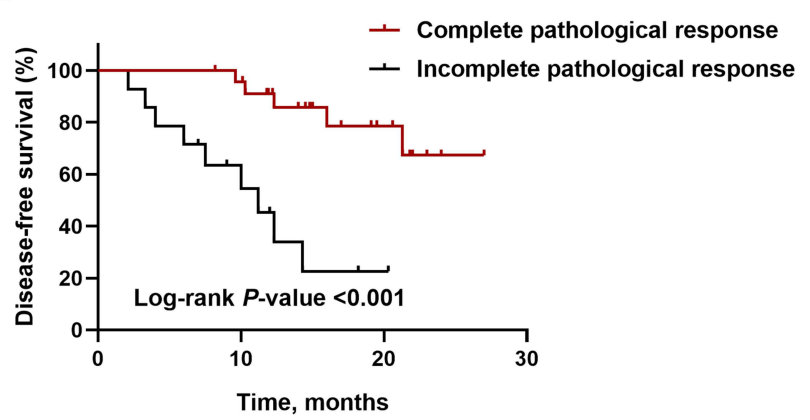

B

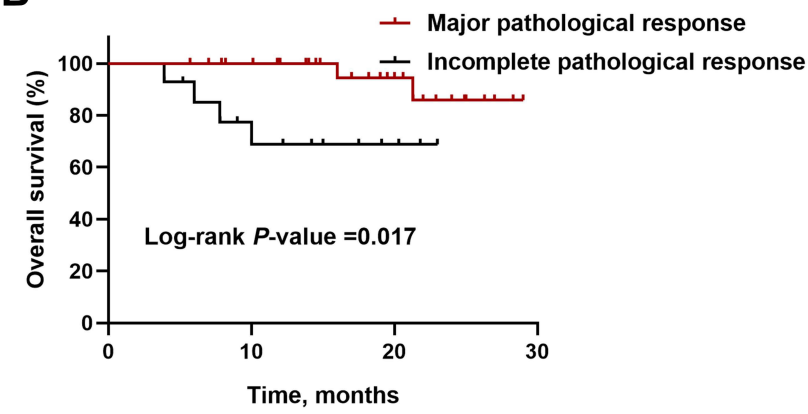

D

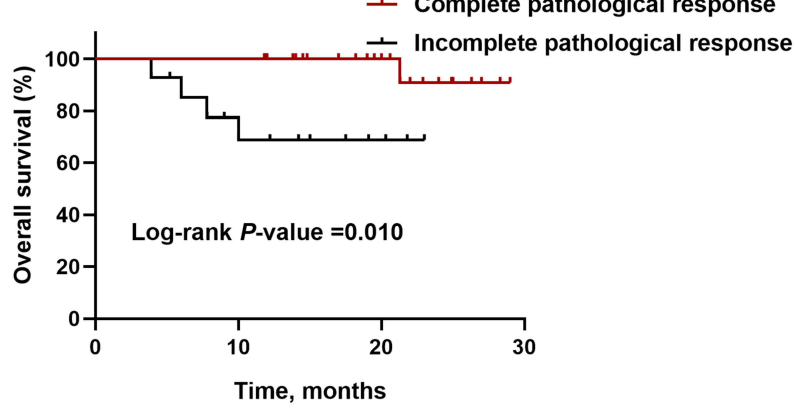

Figure 2 Kaplan-Meier survival curves stratified by pathological responses. (A and B) Disease-free survival (A) and overall survival (B) based on major pathological response. (C and D) Disease-free survival $(\mathbf{C})$ and overall survival (D) based on complete pathological response. 
A
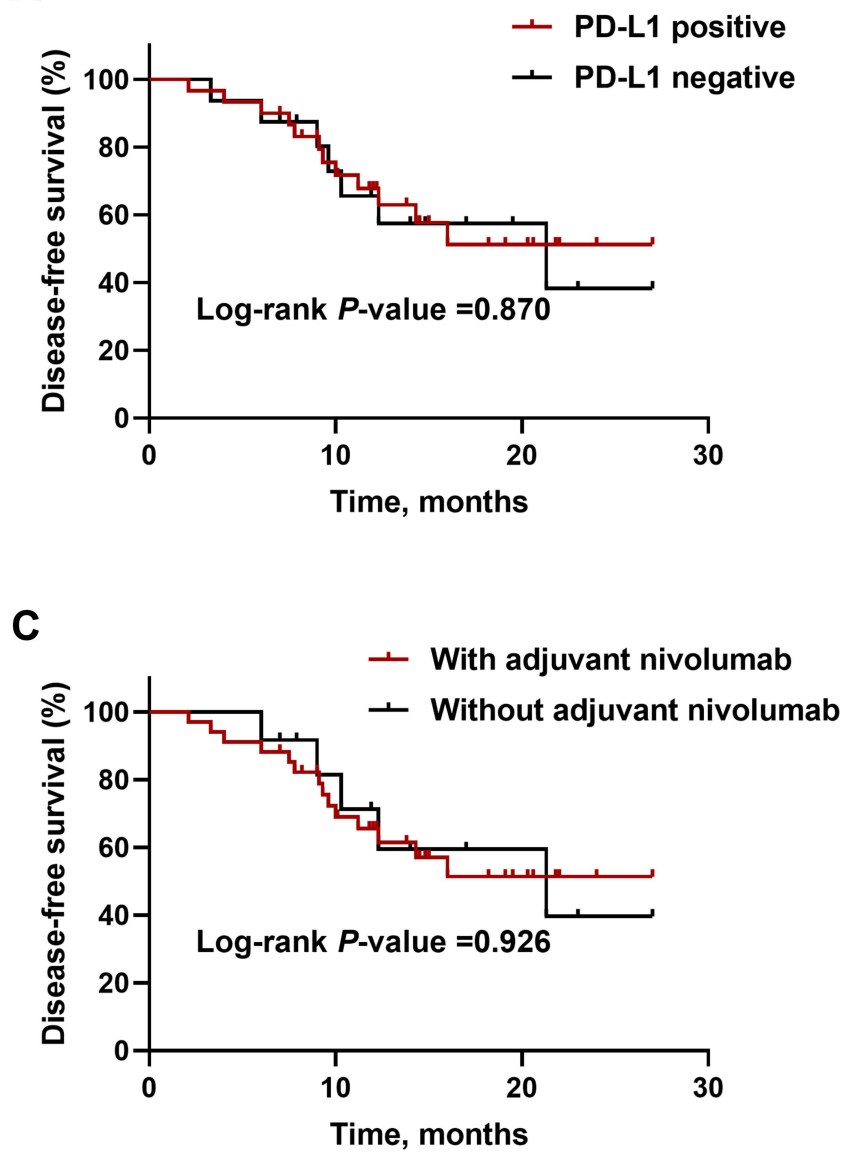

B

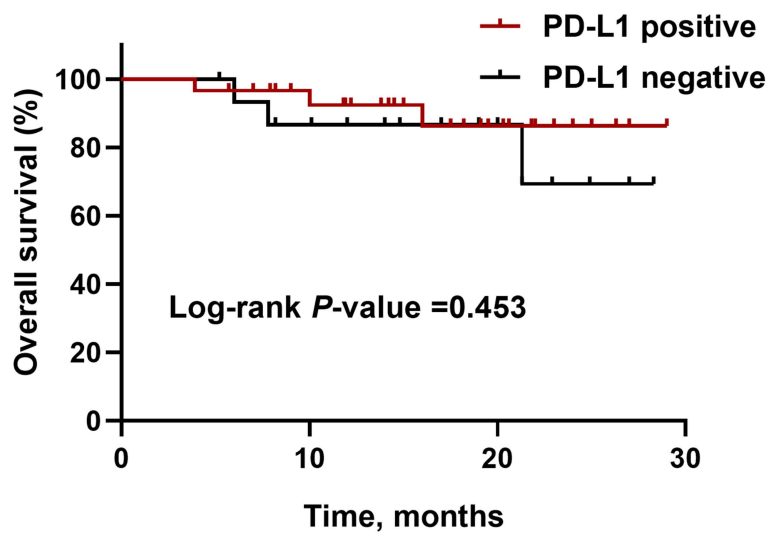

D

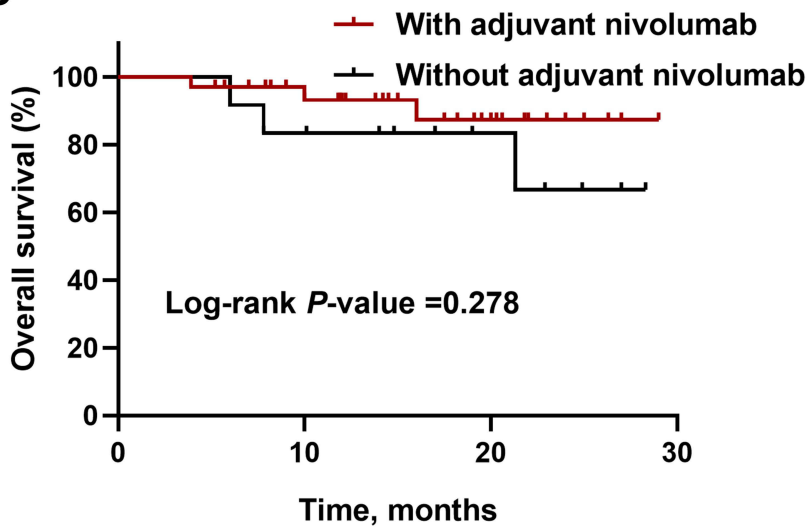

Figure 3 Kaplan-Meier survival curves stratified by PD-LI expression or adjuvant nivolumab. (A and B) Disease-free survival (A) and overall survival (B) based on PD-LI expression. (C and D) Disease-free survival (C) and overall survival (D) based on adjuvant nivolumab.

Patients with stage III NSCLC employ broad anatomic heterogeneity, covering a wide extent of local invasion and nodal involvement. Despite recent improvements in multimodal treatments including neoadjuvant chemotherapy, the survival benefit for patients with NSCLC and nodal disease (N1-N2) was only $4-5 \%,{ }^{21}$ which cast a shadow for management of this population. Recent thriving ICIs have reshaped the neoadjuvant treatments on the theoretical basis that they could induce a stronger adaptive antitumor response and release neoantigens from dying tumor cells before surgical resection. ${ }^{22}$ The recent PACIFIC trial demonstrated the promising efficacy of durvalumab in combination with chemoradiotherapy for patients with locally advanced NSCLC. ${ }^{12}$

Previous concern with neoadjuvant treatment remains the delay of surgery due to disease progression or serious treatment-related AEs during neoadjuvant treatment. In this study, only one patient did not proceed to surgery due to brain metastases, and almost all patients were able to proceed to surgery. Neoadjuvant nivolumab and chemotherapy did not delay planned surgery. In our study, 21 (45.7\%) of 46 patients had multiple level N2 disease, which is considered unresectable by some. Notably, this neoadjuvant chemoimmunotherapy yielded promising results in downstaging with $78.2 \%$ of patients achieving pathological $\mathrm{T}$ downstaging and $65.2 \%$ of patients obtaining pathological $\mathrm{N}$ downstaging. Surgery was planned based on the tumor extension after neoadjuvant chemoimmunotherapy. Our neoadjuvant chemoimmunotherapy could increase the chance of patients who were assumed to be inoperable to have curative surgery. The effect of neoadjuvant chemoimmunotherapy on the technical aspects of lung resection is difficult to quantitatively assess. Commonly used surrogates that are indicative of a difficult operation include blood loss, operative time, hospital stay, and perioperative complications. In this study, blood loss was minimal, operative time and hospital stay were reasonable, 
and the rate of surgical complications was low. The vast majority of patients had R0 resection, which underlines the feasibility and utility of resection in this population.

Early neoadjuvant nivolumab monotherapy trials reported a MPR of $45 \%$ and a pCR of $15 \%$ for patients with resectable early (stage I-IIIA) NSCLC. ${ }^{14}$ Bott et al. also reported a MPR of $45 \%$ and a pCR of $10 \%$ for patients with resectable NSCLC (stage IA-IIIA) receiving resection following neoadjuvant nivolumab. ${ }^{23}$ However, the NEOSTAR trial of nivolumab only showed a MPR of $17 \%$ and a pCR $9 \%$ for patients with stage I-IIIA (single N2) resectable NSCLC. ${ }^{24}$ Comparison with previous studies on induction ICI for NSCLC has been summarized in Table S3 with a MPR of $20-57 \%$ and pCR of $4-63 \%$. In this study, the MPR (17.4\%) and pCR (52.2\%) were impressive for all patients after neoadjuvant chemoimmunotherapy. Moreover, we also showed a favorable survival outcome including DFS and OS. However, the NADIM trial, ${ }^{16}$ which explored the efficacy of neoadjuvant nivolumab combined with standard chemotherapy, acquired better pathological and survival outcomes with a MPR of $20 \%$, a pCR of $63 \%$, and a DFS of $77.1 \%$ and an OS of $89.9 \%$ at 24 months. The main reason for the difference might be that the NADIM trial only included patients with stage IIIA NSCLC, while our study had 16 (34.8\%) patients with stage IIIB NSCLC. Our data on AEs indicate that the neoadjuvant treatment regimens used in this study were well tolerated, with toxic effects similar to data previously reported on paclitaxel and carboplatin. ${ }^{25}$ In addition, immune-related AEs were well monitored and managed, which is consistent with the findings on immunotherapy in a series of studies. ${ }^{18,19,26}$

Subgroup analysis demonstrated that patients with negative PD-L1 expression did not have worse radiological or pathological responses and survival benefit. In another words, patients benefited from neoadjuvant chemoimmunotherapy regardless of PD-L1 expression, which was consistent with the findings of several studies. ${ }^{27}$ Pathological responses including MPR and $\mathrm{pCR}$ were associated with better survival outcomes, which further reinforces the relevance of the pathological response as a potential survival surrogate. In addition, adjuvant nivolumab appeared not to be correlated to survival benefits; however, the results need to be interpreted with caution on the basis of small sample sizes.

There are different neoadjuvant therapies including immunotherapy, chemotherapy, and chemoradiotherapy for locally advanced NSCLC. A meta-analysis showed that neoadjuvant chemotherapy had a significant survival benefit for patients with NSCLC, with a 5-year OS benefit increased by 5\%. ${ }^{6}$ Huang et al. reported a better pathological response in neoadjuvant immunotherapy compared with neoadjuvant chemotherapy in stage III (N2) NSCLC, ${ }^{18}$ with a MPR of $12.8 \%$ and a pCR of $2.6 \%$ in the neoadjuvant chemotherapy group. In terms of neoadjuvant chemoradiotherapy, a retrospective multi-institutional Phase II study compared the pathological responses of neoadjuvant chemotherapy or chemoradiotherapy in stage III (N2) NSCLC, ${ }^{28}$ which reported a MPR of $54.5 \%$ in the tumor and $80 \%$ downstaging in the neoadjuvant chemoradiotherapy group, and $9.5 \%$ and $33.3 \%$ in the neoadjuvant chemotherapy group, respectively. Neoadjuvant immunotherapy or chemoradiotherapy appeared to obtain better pathological responses than neoadjuvant chemotherapy, and more well-designed head-to-head studies should be conducted comparing neoadjuvant immunotherapy and chemoradiotherapy with robust results.

There are several limitations in this research. Firstly, this is a single-center, retrospective study with inherent selection bias, which needs to be validated in multiple centers with more samples. Secondly, the follow-up period was limited, and the 3- or 5-year DFS and OS after neoadjuvant chemoimmunotherapy will be observed in future study.

\section{Conclusions}

In the neoadjuvant setting, nivolumab combined with chemotherapy resulted in favorable survival outcomes, as well as high rates of MPR and pCR, among patients with locally advanced NSCLC. This combination regimen is safe and feasible, which represents a promising therapeutic option for this population.

\section{Data Sharing Statement}

All data generated or analyzed during this study are available upon reasonable request from correspondence author.

\section{Funding}

There is no funding to report. 


\section{Disclosure}

The authors report no conflict of interests in this work.

\section{References}

1. Bade BC, Dela Cruz CS. Lung cancer 2020: epidemiology, etiology, and prevention. Clin Chest Med. 2020;41(1):1-24. doi:10.1016/j. ccm.2019.10.001

2. Boyd JA, Hubbs JL, Kim DW, Hollis D, Marks LB, Kelsey CR. Timing of local and distant failure in resected lung cancer: implications for reported rates of local failure. $J$ Thorac Oncol. 2010;5(2):211-214. doi:10.1097/JTO.0b013e3181c20080

3. Sosa MS, Bragado P, Aguirre-Ghiso JA. Mechanisms of disseminated cancer cell dormancy: an awakening field. Nat Rev Cancer. 2014;14 (9):611-622. doi:10.1038/nrc3793

4. Felip E, Rosell R, Maestre JA, et al. Preoperative chemotherapy plus surgery versus surgery plus adjuvant chemotherapy versus surgery alone in early-stage non-small-cell lung cancer. J Clin Oncol. 2010;28(19):3138-3145. doi:10.1200/JCO.2009.27.6204

5. Zhu J, Zhang Y, Wang M, et al. Outcomes in 36 patients with stage IIIA-N2 squamous cell carcinoma of the lung treated with nab-paclitaxel plus carboplatin as neoadjuvant therapy: a Prospective Study from a single center. Med Sci Monit. 2021;27:e930738. doi:10.12659/MSM.930738

6. Group NM. Preoperative chemotherapy for non-small-cell lung cancer: a systematic review and meta-analysis of individual participant data. Lancet. 2014;383(9928):1561-1571. doi:10.1016/S0140-6736(13)62159-5

7. Borghaei H, Gettinger S, Vokes EE, et al. Five-year outcomes from the randomized, Phase III trials CheckMate 017 and 057: nivolumab versus docetaxel in previously treated non-small-cell lung cancer. J Clin Oncol. 2021;39(7):723-733. doi:10.1200/JCO.20.01605

8. Herbst RS, Garon EB, Kim DW, et al. Long-term outcomes and retreatment among patients with previously treated, programmed death-ligand 1positive, advanced non-small-cell lung cancer in the KEYNOTE-010 Study. J Clin Oncol. 2020;38(14):1580-1590. doi:10.1200/JCO.19.02446

9. Paz-Ares L, Vicente D, Tafreshi A, et al. A randomized, placebo-controlled trial of pembrolizumab plus chemotherapy in patients with metastatic squamous NSCLC: protocol-specified final analysis of KEYNOTE-407. J Thorac Oncol. 2020;15(10):1657-1669. doi:10.1016/j.jtho.2020.06.015

10. Garassino MC, Gadgeel S, Esteban E, et al. Patient-reported outcomes following pembrolizumab or placebo plus pemetrexed and platinum in patients with previously untreated, metastatic, non-squamous non-small-cell lung cancer (KEYNOTE-189): a multicentre, double-blind, randomised, placebo-controlled, Phase 3 trial. Lancet Oncol. 2020;21(3):387-397.

11. Gandhi L, Rodríguez-Abreu D, Gadgeel S, et al. Pembrolizumab plus chemotherapy in metastatic non-small-cell lung cancer. $N$ Engl $J$ Med. 2018;378(22):2078-2092. doi:10.1056/NEJMoa1801005

12. Antonia SJ, Villegas A, Daniel D, et al. Durvalumab after chemoradiotherapy in stage III non-small-cell lung cancer. N Engl J Med. 2017;377 (20):1919-1929. doi:10.1056/NEJMoa1709937

13. Rizvi NA, Hellmann MD, Brahmer JR, et al. Nivolumab in combination with platinum-based doublet chemotherapy for first-line treatment of advanced non-small-cell lung cancer. J Clin Oncol. 2016;34(25):2969-2979. doi:10.1200/JCO.2016.66.9861

14. Forde PM, Chaft JE, Smith KN, et al. Neoadjuvant PD-1 blockade in resectable lung cancer. N Engl J Med. 2018;378(21):1976-1986. doi:10.1056/ NEJMoa1716078

15. Gao S, Li N, Gao S, et al. Neoadjuvant PD-1 inhibitor (Sintilimab) in NSCLC. J Thorac Oncol. 2020;15(5):816-826. doi:10.1016/j. jtho.2020.01.017

16. Provencio M, Nadal E, Insa A, et al. Neoadjuvant chemotherapy and nivolumab in resectable non-small-cell lung cancer (NADIM): an open-label, multicentre, single-arm, Phase 2 trial. Lancet Oncol. 2020;21(11):1413-1422. doi:10.1016/S1470-2045(20)30453-8

17. Chen T, Ning J, Campisi A, et al. Neoadjuvant PD-1 inhibitors and chemotherapy for locally advanced NSCLC: a Retrospective Study. Ann Thorac Surg. 2021. doi:10.1016/j.athoracsur.2021.03.041

18. Huang Z, Wu Z, Qin Y, et al. Perioperative safety and feasibility outcomes of stage IIIA-N2 non-small cell lung cancer following neoadjuvant immunotherapy or neoadjuvant chemotherapy: a retrospective study. Ann Transl Med. 2021;9(8):685. doi:10.21037/atm-21-1141

19. Duan H, Wang T, Luo Z, et al. Neoadjuvant programmed cell death protein 1 inhibitors combined with chemotherapy in resectable non-small cell lung cancer: an open-label, multicenter, single-arm study. Transl Lung Cancer Res. 2021;10(2):1020-1028. doi:10.21037/tlcr-21-130

20. Detterbeck FC, Boffa DJ, Kim AW, Tanoue LT. The eighth edition lung cancer stage classification. Chest. 2017;151(1):193-203. doi:10.1016/j. chest.2016.10.010

21. Hanna GG, Siva S, Plumridge N, Solomon B, Ball DL. Preoperative chemotherapy for non-small-cell lung cancer. Lancet. 2014;384 (9939):232-233. doi:10.1016/S0140-6736(14)61208-3

22. Liu J, Blake SJ, Yong MC, et al. Improved efficacy of neoadjuvant compared to adjuvant immunotherapy to eradicate metastatic disease. Cancer Discov. 2016;6(12):1382-1399. doi:10.1158/2159-8290.CD-16-0577

23. Bott MJ, Yang SC, Park BJ, et al. Initial results of pulmonary resection after neoadjuvant nivolumab in patients with resectable non-small cell lung cancer. J Thorac Cardiovasc Surg. 2019;158(1):269-276. doi:10.1016/j.jtcvs.2018.11.124

24. Cascone T, William WN, Weissferdt A, Lin HY, Sepesi B. Neoadjuvant nivolumab (N) or nivolumab plus ipilimumab (NI) for resectable non-small cell lung cancer (NSCLC): clinical and correlative results from the NEOSTAR study. J Clin Oncol. 2019;37(15_suppl):8504. doi:10.1200/ JCO.2019.37.15_suppl.8504

25. Socinski MA, Bondarenko I, Karaseva NA, et al. Weekly nab-paclitaxel in combination with carboplatin versus solvent-based paclitaxel plus carboplatin as first-line therapy in patients with advanced non-small-cell lung cancer: final results of a phase III trial. J Clin Oncol. 2012;30 (17):2055-2062. doi:10.1200/JCO.2011.39.5848

26. Shu CA, Gainor JF, Awad MM, et al. Neoadjuvant atezolizumab and chemotherapy in patients with resectable non-small-cell lung cancer: an open-label, multicentre, single-arm, phase 2 trial. Lancet Oncol. 2020;21(6):786-795. doi:10.1016/S1470-2045(20)30140-6

27. Chen Y, Zhou Y, Tang L, et al. Immune-checkpoint inhibitors as the first line treatment of advanced non-small cell lung cancer: a meta-analysis of randomized controlled trials. J Cancer. 2019;10(25):6261-6268. doi:10.7150/jca.34677

28. Muñoz-Guglielmetti D, Sanchez-Lorente D, Reyes R, et al. Pathological response to neoadjuvant therapy with chemotherapy vs chemoradiotherapy in stage III NSCLC-contribution of IASLC recommendations. World J Clin Oncol. 2021;12(11):1047-1063. doi:10.5306/wjco.v12.i11.1047 


\section{Publish your work in this journal}

Cancer Management and Research is an international, peer-reviewed open access journal focusing on cancer research and the optimal use of preventative and integrated treatment interventions to achieve improved outcomes, enhanced survival and quality of life for the cancer patient. The manuscript management system is completely online and includes a very quick and fair peer-review system, which is all easy to use. Visit http://www.dovepress.com/testimonials.php to read real quotes from published authors.

Submit your manuscript here: https://www.dovepress.com/cancer-management-and-research-journal 